\title{
Unintentional entrainment effect in a context of Human Robot Interaction: an experimental study
}

\author{
Eva Ansermin ${ }^{1}$, Ghiles Mostafaoui ${ }^{1}$, Xavier Sargentini ${ }^{1}$ and Philippe Gaussier ${ }^{1}$
}

\begin{abstract}
Modelling nonverbal communication in robotics is a crucial issue to improve Human Robot interactions (HRI). Among several nonverbal behaviours we focus in this article on unintentional rhythmic entrainment and synchronization which has been proven to be highly important in intuitive and natural Human Human communication. Hence, the rising question is whether or no this phenomenon can be reproduced in a context of $\mathrm{HRI}$ and what are the prerequisites to ensure its emergence. In this paper, we study rhythmical interactions during imitation games between a NAO robot and naive subjects. We analysed two main types of interactions, a first where NAO performs movements at a fixed rhythm (unidirectional) and a second one where the robot is able to adopt the human motion dynamic (bidirectional) using a neural modelling of the entrainment effect based on dynamical systems. We show that using such model allows us to reach synchronization during the interactions and that both partners (robot and human) adapt their frequency as observed in natural HHI. This puts forward the importance of bidirectionality for HRI. Moreover, the participants shifted their motion dynamics during the interaction without noticing it, proving the presence of such unintentional rhythmic entrainment in HRI.
\end{abstract}

Index Terms - Synchronisation, Entrainment effect, Human Robot Interaction, bidirectional interactions, neural network, bio-inspired robotic.

\section{INTRODUCTION}

The emergence of a growing number of applications involving robots in our daily life brings the outbreak of numerous problematic concerning social robotics. It becomes clear that research in robotics should not only focus on the performance of a robot in specific tasks, but also in the manner to integrate robots in a human environment to perform satisfying and intuitive Human Robot Interaction (HRI). In 2012, Mortl et al. showed the importance of the robot adaptation capacities during physical cooperative tasks; performances are increased when the robot reacts according to the human dynamic feedback [1]. This highlights the fact that human and robot should cooperate and adapt during a task like we do during classical Human Human Interactions (HHI). In HHI, such cooperation does not imply only explicit behaviour such as speech or gesture, but also several important nonverbal behaviours that play a key role in communication. It has been proven that using nonverbal communication in HRI can

This research was supported by the DGA and the Agence Nationale de la Recherche grant (DIRAC Project ANR 13-ASTR00188-01

${ }^{1}$ ENSEA, University of Cergy-Pontoise, UMR CNRS 8051 ETIS, FRANCE. increase the robot's perceived anthropomorphism and likeability [2]. The interaction is more natural and the human can relate to the robot. Moreover, as a more pragmatic argument, Breazeal et al. showed that nonverbal communication has a direct impact on the performance during a task with a robot [3] and allows the system to be more robust to miscommunication.

An important nonverbal behaviour in HHI is unintentional synchronization and rhythmic entrainment phenomenon. Synchronization is a particular state where two signals or occurrences have the same frequency and are in phase or in opposition of phase. Synchrony is often present in nature at different levels such as mechanic (synchronization of pendulums [4]) and biology (birds in flight, firefly twinkling [5]). This state can also be observed between interacting humans [6]. In fact, our movements can be set into motion and their frequency influenced by external stimuli (visual, audio or tactile), resulting in synchronizations with the stimuli signals. This can be observed at a daily basis as for walking side by side (step synchronization) or a clapping audience [7]. Not only this phenomenon is spontaneous, it is also inevitable even if we explicitly ask people not to synchronize with others, as shown by Issartel et al. [6]. Because of this irrepressible aspect, this can be extended to social interaction, during which healthy partners tend to regularly reach phase of synchronization (saccadic eyes, hands movements) and consequently be coordinated [8]. Furthermore, it has been proven by studying numerous kinds of $\mathrm{HHI}$ in different contexts, that synchronization is linked to the degree of partners attention, making it an important unintentional signal for social interactions [9] [10] [11]. Numerous other examples showing the importance of synchronization and coordination in nonverbal behaviour can be cited, it has been shown that: a baby needs the mother to be synchronized to better interact with her [12], learning is enhanced when the teacher is in phase with his student [13], the more we find people attractive, the more we synchronize with them [14], etc. Such phenomenon have also be reported during HRI. For example, Ogawa et al. reported an entrainment of nodding between a human and a robot [15] and Sakamaoto et al showed a person's body movements are entrained to robot's ones in the situation of a route guide[16].

We can distinguish two types of synchronization: intentional synchronization and unintentional ones. The main difference is that intentional synchronization requires the will of the subject to coordinate whereas unintentional synchronization occurs without the subject noticing. In 2012, Varlet et al. showed that schizophrenia impacts intentional synchronization whereas the capacity of unintentional 
synchronization remains intact [17]. This suggests that intentional and unintentional synchronization may not be a part of the same process. In fact, unintentional synchronization seems an automatic process, it may require less cognitive abilities than intentional synchronization. It is admitted, in the literature, that subjects can be unintentionally synchronized when the external rhythmical stimuli is around $10 \%$ of their own proper frequency. It is worth noticing that, when we mention the importance of coordination during conversation and interaction, we mainly speak about unintentional synchronization that occurs during those exchanges.

When facing a rhythmic stimulus, one can be unintentionally synchronized if his frequency is closed enough. However, when it's not the case, the movement is still influenced and tend to be closer to the rhythmic signal even if not in a stable state and not synchronized; this phenomenon, tightly linked to synchrony is called entrainment effect or magnet effect. As for synchrony, the entrainment effect is spontaneous and cannot even be avoided [8]. A strong hypothesis is that unintentional synchronization may be a consequence, a stable state, of a bidirectional (mutual) entrainment effect.

The above state of the art highlights the importance of unintentional rhythmic adaptation and synchronizations in HHI. It would be consequently interesting to study these phenomenon in HRI. Indeed, synchronization permits to ease attention and concentration [18] [8]. On the contrary, non-synchronized rhythm can be disturbing during a task [19] and even negative in a psychological approach as shown by Lumsden et al. [20]. One can augur that this phenomenon could be exploited in social robotics in order to make HRI more natural. It implies making the robot able not only to adapt its behaviour to the human dynamics but also to induce unintentional rhythmic adaptations of the partner's movements. In fact, Lorentz et and colleagues [21] proved that humans do not take over the complete effort of movement adaptation to reach synchronization when facing a moving robot that doesn't adapt its rhythm. This shows that the bidirectionality is crucial and that we need adaptive robots.

In the line of this state of the art, we will study, in this paper, this phenomenon of unintentional synchronization and rhythmic entrainment in HRI. More precisely, we will embed a NAO robot with a neural model permitting rhythmical entrainment and adaptation (to human movements) and conduct HRI experiments with naive subjects in order to validate our model and show if it can induce, as in HHI, unintentional or unintentional human rhythmic adaptation.

\section{Modelling RHYTHMiC ENTRAinMENT}

As stated before, studying unintentional synchronization and rhythmic entrainment in HRI implies first making the robot able to be rhythmically entrained by the human's motion dynamics. Several approaches can then be considered. From an ecological point of view, recent studies put forward milliseconds inter-brain synchronization during a social task [22]. Such timing shows that this process is very low level and may come from a self-organized system and not from a complex computational process. This vision of the emergence of synchronized systems is highly defended by dynamical systems theories. In such approaches, we model the agent's dynamics by oscillators influencing and entraining rhythmically each other (as Hugen's pendulums). As a simple example, two pendulums (at different frequencies) on the same table can synchronize because of the vibration of this table which permit an energy exchange between the two systems. Pantaleone linked those mechanical synchronization to biological ones [4]. Haken Kelso and Bunz proposed a system of equations modelling the behaviour of coupled Van Der Pol oscillators [23]. By using dynamical systems, we consider that agents bring energy to each other via stimuli (visual for example) to entrain the partner's rhythmic behaviour in a very low level process and consequently change their mutual frequency and motion amplitude.

Modelling two systems (agents) rhythmically influencing each other using dynamical systems implies the use of coupled oscillators, whether they are mathematical [23] or neural [24]. A major difficulty in HRI is the fact that we don't have a direct access to both agents" "oscillators" (human motion frequency and phase for example). To tackle this problem, we will use here the entrainment effect model we recently proposed [25] (see figure 1). This model has been validated with naive subjects and has been proven to be efficient to adapt the robot's rhythm to the human motion dynamics in a very simple HRI task. However, it has not been tested on different moves and tasks.

For clarity sake, this model will be explained briefly below but we invite the reader to refer to [25] for more details.

The model uses a neural oscillator to control the robot rhythmical movement whose dynamic can be influenced by an energy coming from the optical flow induced by the human motion. The global model is illustrated figure 1

Figure 1 shows a neural oscillator inspired by the Prepin model [26]. The equations respected by our neurons are given by equation 1 and 2

$$
\begin{aligned}
& N_{1}(n+1)=N_{1}(n)-\beta N_{2}(n)+\alpha 1 \\
& N_{2}(n+1)=N_{1}(n)+\beta N_{2}(n)+\alpha 2
\end{aligned}
$$

The output of both neurons is consequently oscillatory and the frequency depends on the parameter $\beta$.

This oscillator controls the robot's arm and can be influenced by the movement of an interacting partner. A camera is located in front of the robot. The optical flow is extracted from the images by a hierarchical algorithm proposed by Amiaz et al. [27]. The optical flow is then used to obtain directional neurons sensitive to vertical or horizontal movements. These neurons provide a periodical response when the movement is rhythmic which can alternates between negatives and positives values.

$$
N 1(n+1)=N 1(n)-\beta N 2(n)-\alpha 1+c p * f(n)
$$

This signal $f$ is then modulated by a coupling factor $c p$ and added to the oscillator signal (see equation 3). This added signal changes the dynamic of the oscillator and can lead to synchronization with the partner. The coupling factor $c p$ allows us to weight the amplitude of the input signal and so the range of frequencies in which the oscillator would be able 
to be synchronized; if the coupling factor is high, the oscillator will be able to synchronized in a large range of frequencies and vice versa. Figure 2 shows the simulated resulting entrainment of the oscillator for two different coupling factors. We can observe that, for a small coupling factor (0.05), the oscillator is influenced and its dynamic is deformed but is not at the same frequency as the simulated optical flow induced by human motion. On the contrary we can see that a higher coupling factor (0.2) allows the oscillator to have the same frequency as the simulated optical flow signal. Precisely, we can see that those two signals are out of phase with a shift of $\pi / 2$. The optical flow signal being the derivative of the human arm position, this means that the oscillator is indeed synchronized with the human arm movement.

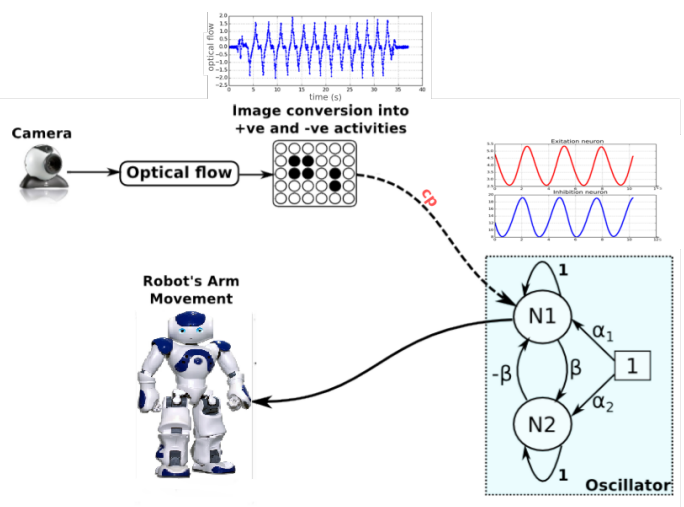

Fig. 1: The entrainment model used during the experiments
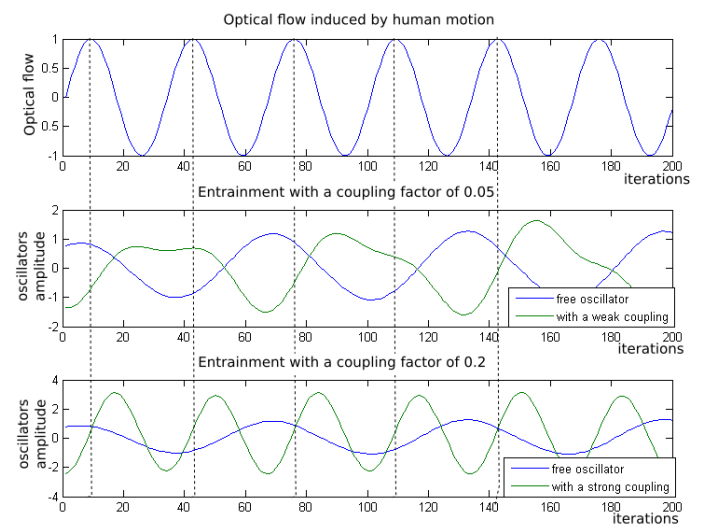

Fig. 2: Results on the Same rhythmic entrainment of the oscillator for two different coupling factors

\section{STUdying UNINTENTIONAL RHYTHMIC ENTRAINMENT IN HRI}

We performed experiments for studying the visual entrainment effect in HRI during imitation games between a NAO robot and naive subjects. Two main types of interaction were analysed $:$ i) unidirectional : the robot moves with a fixed frequency of $0.73 \mathrm{~Hz}$ without taking into account the human motion dynamic ii) bidirectional : the robot is able to be adaptive to the human rhythm using the previously presented neural model.

\section{A. Experimental set up}

Each participant stands facing the NAO robot about 1.5 meter in distance. The robot was seated on a small chair on a table and at the same height as the human partner (see figure 3). The robot can perceive the human partner through an external camera to avoid the limitations of the NAO's one. The collected data (the oscillator controlling the robots arm and the optical flow induced by the human movements) are saved directly during the experiments.

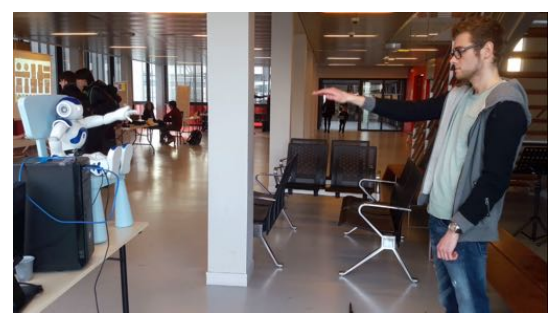

Fig. 3: Experimental set up

1) Subjects: The experiments were conducted on nine young healthy adults. Two women and seven males.

2) Condition: For studying the human unintentional entrainment during the HRI, the real goal of the experiments was hidden from the participants, they were told that the objective was to build a data base which would be used by the robot to learn to recognize different gestures. They had to make three different rhythmical movements that were in a random order. It is important to note that they were explicitly asked to move naturally with their own preferred frequency regardless to the robot behaviour.

We studied three rhythmical movements:

- (1) raising and lowering the arms vertically (mov1)

- (2) raising and lowering the arm horizontally(mov2)

- (3) waving at the human (mov3)

Video showing NAO producing those movement and a practical example of the entrainment model are available at this link 1 .

Each subject performs the three movements in three different conditions:

- First condition: the purpose of this part is to obtain the proper frequency of the participants for each movement. We explain the subjects the movement and ask them to do it alone at their own preferred rhythm. Each movement is done for 30 seconds in a random order.

- Second condition: here, we study the influence of the robot which moves at its own pace (without entrainment or adaptation). Consequently we study the entrainment effect in a unidirectional context. The robot is just an automaton and is not influenced by the rhythm of the human subject. We ask the subject to do the same movement as the robot but still at their own pace as in the first condition. The robot's purpose is just to show them which movement is to be done. The three movements are made in a random order, each during 30 seconds.

\footnotetext{
${ }^{1}$ www.etis.ensea.fr/neurocyber/Videos /authors/ansermin/RoMan2017
} 
- Third condition: this part concerns the study of entrainment effect in a bidirectional interaction. In this case, the robot is able to be entrained by the partner thanks to the model presented in the previous section. We fixed the coupling factor to a value of 0.1 . As previously, the subject was asked to do the same movement as the robot at their own rhythm and the three movements were ordered randomly.

3) Hypothesis: We expect to obtain, in the second condition, an entrainment effect on the human movement and eventually synchronizations if the robot and the partner's preferred frequency are close enough (around 10\% if we consider the data obtained for $\mathbf{H H I}$ in the literature). This would allow to put forward and analyses the entrainment effect caused by the robot. For the third condition, we expect to find a large majority of cases where the robot and the human are synchronized which would occur, due to the mutual rhythmic entrainment, at a frequency between the robot's original one $(0.73 \mathrm{~Hz})$ and the human's preferred frequency (given by the first condition).

\section{ExPERIMENTAL Results}

Because we studied the entrainment effect with two different kinds of interaction (with and without adaptation of the robot), we will present separately the results of the condition 2 and 3 . We remind the reader that the first condition is only used to extract the preferred frequency of each subject performing each of the three studied movements.

\section{A. Condition 2: unidirectionnal entrainment}

In this section we compare the subject's proper frequency (retrieved during the first condition) with his frequency while interacting with the robot during the second condition. The robots moves at a frequency of $0.73 \mathrm{~Hz}$. The figure 4 shows a general view of the results for all the subjects.

As we can see in the graphics, during the second condition every participant has been influenced by the robot's motion; the gap between their proper frequency and the robot's one is reduced when the robot moves in front of them. This highlights the existence of the entrainment effect as well as its omnipresence. As mentioned in the protocol, we explicitly asked the participant to keep their own pace and, still, we could observe an important difference of frequency between the first and the second condition, from $4 \%$ to $50 \%$ difference.

Figure 5 studies the entrainment effect according to the difference between the proper frequency of the robot and the human. For that purpose, we plotted the drift of frequency between the first and second condition (the entrainment) as a function of the gap between the proper frequencies. Cases of synchronisation can be observed when the point are on the unitary line or very close. During this second condition, we found 9 cases (trials) of synchronization between the robot and the subjects. We can take note that this usually corresponds to the subjects having proper frequencies (in condition 1) close to the robot's one $(0.73 \mathrm{~Hz})$. In particular, the four cases where the subject proper frequency was in a range of $10 \%$ from the robot were all synchronized during the second condition which is in the line of the results obtained for unintentional synchronization in Human

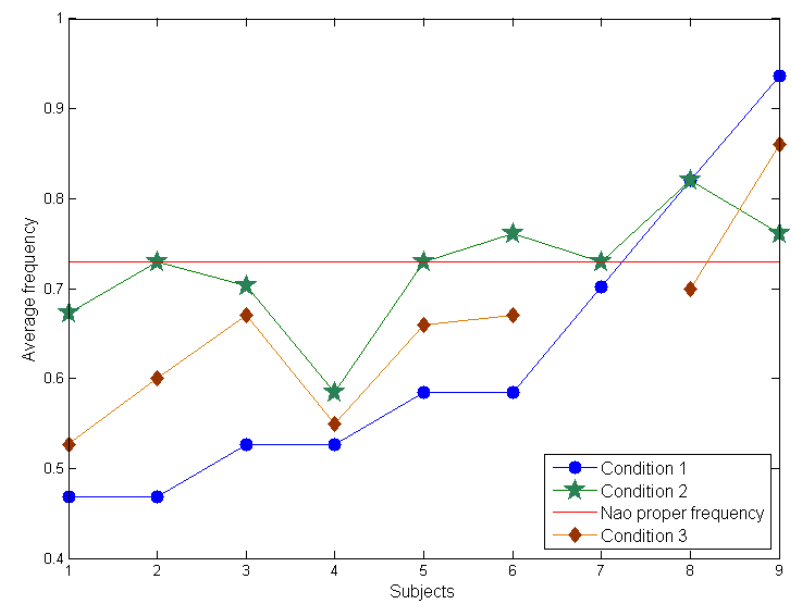

(a) Frequencies during the three conditions for mov1

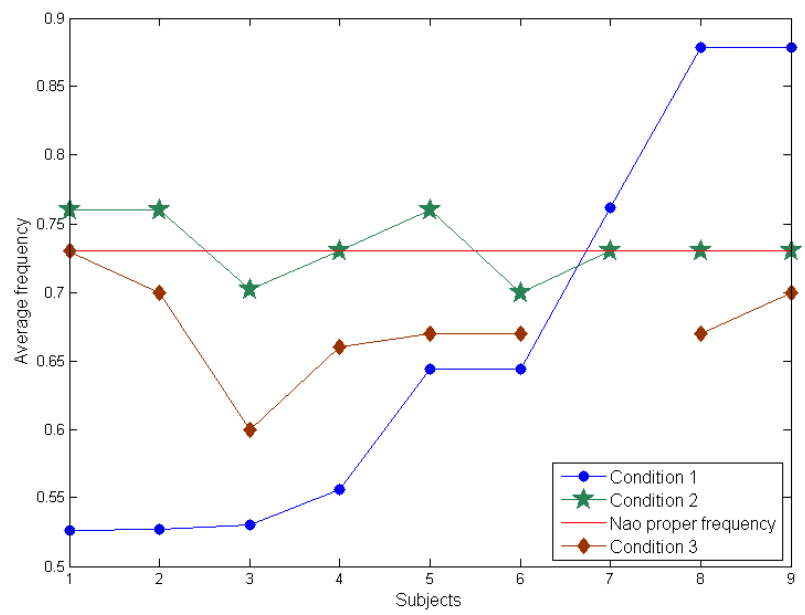

(b) Frequencies during the three conditions for mov2

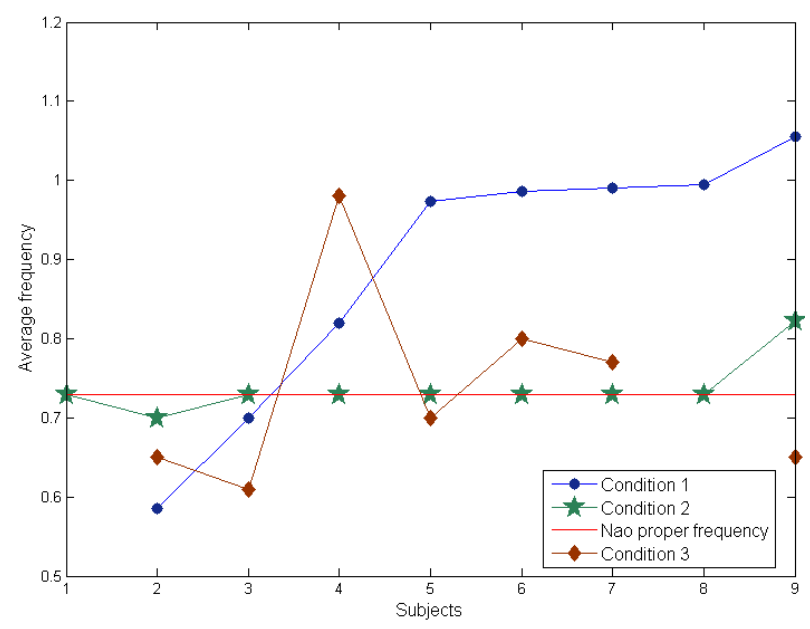

(c) Frequencies during the three conditions for mov3

Fig. 4: Average motion frequencies while performing the three interactive movements during at the three different tasks conditions for all the movement 
Human Interaction. An interesting result is that the gap frequency between the two condition are closer to the unity function when the proper frequency are closer.

The average range between the proper frequency (condition 1) of the subjects and their frequency during this unidirectional interaction is around $23 \%$ which is higher than what we expected (around $10 \%$ as in HHI). This could imply the presence of a strong, and not expected, coupling (visual but also audio because to the robot's motors noise) between the human and NAO. A possible explanation can be the lack of an explicit intentional task hiding the unintentional one (rhythmic adaptation) during our study. It is in fact hard to ensure that some subjects didn't understand the "rules" and were trying to synchronize intentionally with the robot during some trials. To validate this theory, we ignored cases of synchronization during the second condition: the resulted gaps were even more important (36\%) invalidating this explanation. As in a majority of cases, the subject and the robot weren't synchronized, we can assume that this phenomenon is indeed unintended.

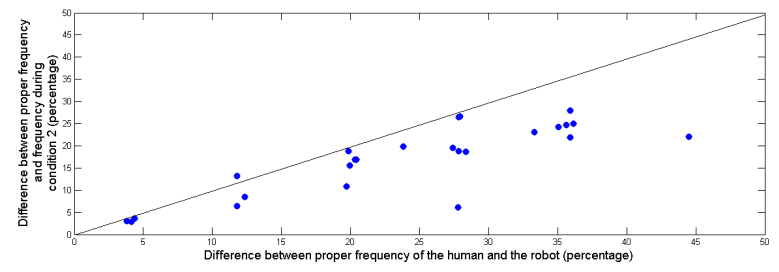

Fig. 5: Frequency gap between condition 1 and 2 for the human (percent) according to the difference of proper frequency (percent). The line represents $y=x$.

Another interesting result is that the entrainment is not the same according to the movement we consider; indeed if we consider only the synchronized cases during this second condition, we notice that the third movement (mov3) is the one where we can find the most case of synchrony. Considering only the cases of synchrony, it is the movement corresponding to the most important entrainment $19 \%$. We wonder if this is the case because this movement (waving, saying hello) is particularly social.

\section{B. Condition 3: Bidirectional entrainment}

During this condition, we use the previously detailed entrainment model, the robot is here able to be adaptive and can be influenced by the subject. In this interaction, the entrainment is bidirectional.

Figure 4 shows all the average motion frequencies during this third condition. During the interactions, synchronization was reached for $100 \%$ of the cases. An example is presented figure 7. During this condition, even if the robot was able to be entrained by the human motion, we were limited to a range of around $20 \%$ for possible synchronizations. Beside, we can observe that the interaction synchronous frequency is never the subject proper frequency, meaning that the human was influenced as well as the robot. As a matter of fact, we note that $75 \%$ of the interaction frequencies were located between the own frequency of the robot and the subject ones; the rhythmic adaptation came from both agents. This can be observed in figure 4 where we can clearly see that the frequency of the third condition is located between the two other curves (conditions 1 and 2). Figure 6 shows the entrainment in condition 3 (difference between frequency for condition 1 and 3 ) in function of the difference between the proper frequency. Comparing to the figure 5, we can observe that, indeed, the entrainment is less important thanks to the robot's on adaptation. In average, we have an entrainment in average of $19 \%$ and an important number of cases around $15 \%$. This condition shows the symmetric role of the human and the robot and the necessity for both side to be entrained to reach synchronization.

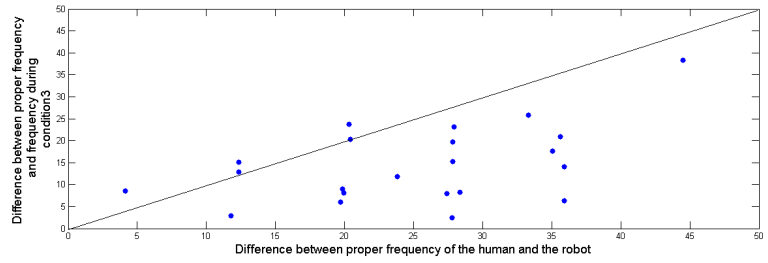

Fig. 6: Frequency gap between condition 1 and 3 for the human (percent) according to the difference of proper frequency (percent). The line represents $y=x$.

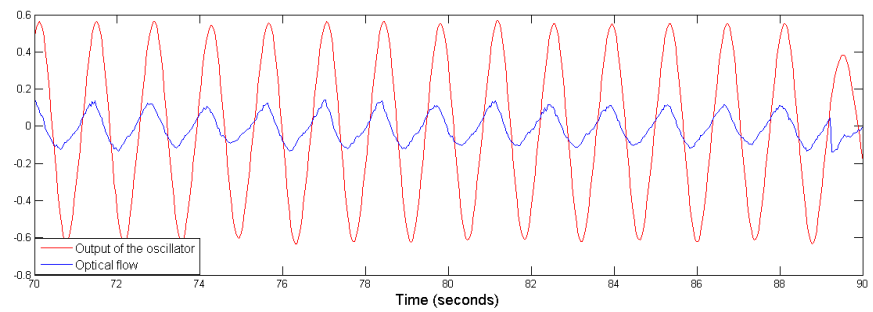

Fig. 7: Example of the optical flow of the subject (blue) and the position of the robot's hand (red).

\section{Analysing the relative phase}

Another interesting information is the relative phase between the robot and the human. Indeed, as we mentioned before, synchronization does not only imply interacting at the same frequency but also being in phase or in opposition of phase.

The figures 8 and 9 represent, respectively for the conditions 2 and 3 , the histogram of the average relative phase between the robot's oscillator and the human motion (extracted by the optical flow). One can notice that the different average relative phases are largely more diffuse for the condition 2 . On the contrary, the histogram for the third condition shows that a majority of the mean relative phases are close to zero or $\pi$ (with a small delay because of the temporal latency induced by our measurement. We can notice that the histogram of the second condition shows a maximum around $\pi$. The reason is that we have (as mentioned in the previous section) 9 cases (trials) of anti-phase synchronizations during this condition. It is interesting to notice that, in this specific condition, every case of synchronized interaction is in opposition of phase whereas in the third condition (bidirectional), a majority are in phase. We wonder if this phenomenon occurred because we asked the participant to keep moving with a rhythm that is natural for them, they probably wanted to consciously 
avoid synchronization and, as the robot is not adaptive (unidirectional), they ended synchronized in opposition of phase because of the unintentional entrainment. Studying this phenomenon more precisely in HRI could be an interesting short-term objective.

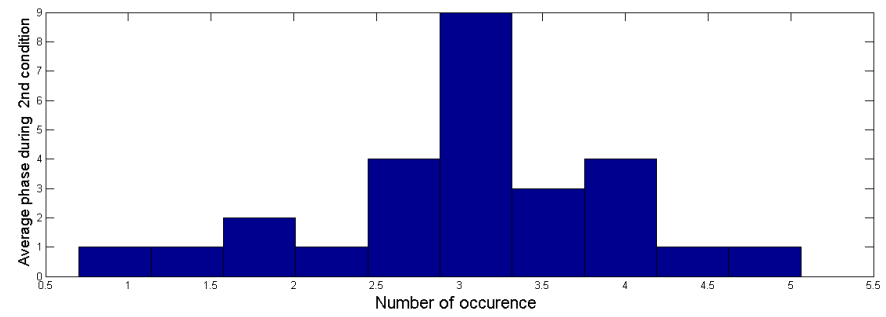

Fig. 8: Histogram of average relative phase during condition 2

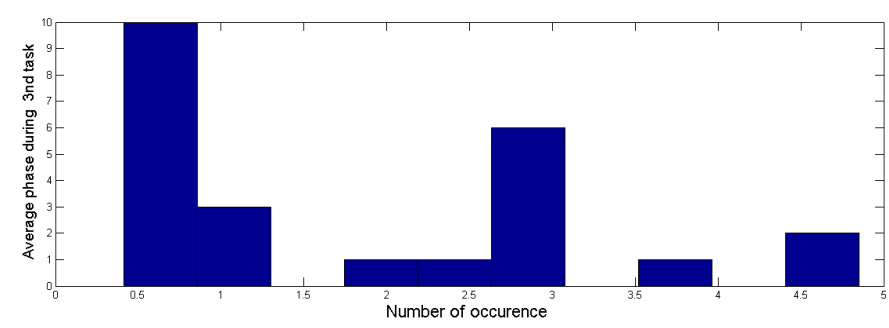

Fig. 9: Histogram of average relative phase during condition 3

\section{Conclusion And Discussion}

Our study shows that unintentional rhythmic entrainment and synchronization can be present during HRI. The condition 2 of our experiments highlights the unintentional entrainment effect caused by a robot moving at a fixed rhythm and perceived by the human partner. As for HHI, we also demonstrated in this condition that if the human natural frequency is close to the robot's one, he can not avoid unintentional entrainment leading to a synchronized interaction. However, we found out that this entrainment was more important $(23 \%)$ than the usual entrainment admitted by the state of arts regarding HHI (10\%). Investigating this particular question about the entrainment effect in HRI, and comparing it with $\mathrm{HHI}$, is in our near objectives.

Another interesting point is that using a robot able to be entrained as well (with an entrainment model based on dynamical systems) allows us to show the importance of bidirectional influence to reach synchronization during an interaction. This is even more proven by the fact that the frequency of the interaction is located between the proper frequency of the robot and the human natural (preferred) one, meaning that both of them has changed their frequency closer to their partner's one. This means that we need rhythmically adaptive robots to obtain a natural coordinated interaction.

Hence, as an extension of this work we need to study these unintentional rhythmic entrainment (in HRI) in a more complex and ecological, cooperative tasks. A more substantial statistical study must also be done to confirm the preliminary results we obtained here and the influence of rhythmically adaptive robots on the performance during such task.

Another important perspective is the interest of using robots able to simulate and to induce unintentional rhythmic adaptation to help understanding unintentional human behaviour. In fact, being able to control important parameters (such as the proper frequency of the robot, the phase, the coupling factor etc.) makes the robot a useful tool to study unintentional human adaptations in different experimental conditions. Moreover, these type of studies can actually find interesting applications in medical or clinical rehabilitation therapies for impaired people, specifically the ones suffering from social or sensorimotor disorder (schizophrenia, autism, Parkinsonian etc.).

\section{REFERENCES}

[1] Alexander Mörtl, Martin Lawitzky, Ayse Kucukyilmaz, Metin Sezgin, Cagatay Basdogan, and Sandra Hirche. The role of roles: Physical cooperation between humans and robots. The International Journal of Robotics Research, 31(13):1656-1674, 2012.

[2] Maha Salem, Friederike Anne Eyssel, Katharina Rohlfing, Stefan Kopp, and Frank Joublin. To err is human (-like): Effects of robot gesture on perceived anthropomorphism and likeability. International Journal of Social Robotics, 5:313-323, 2013.

[3] Cynthia Breazeal, Cory D. Kidd, Andrea Lockerd Thomaz, and Guy Hoffmanand Matt Berlin. Effects of nonverbal communication on efficiency and robustness in human-robot teamwork. In Intelligent Robots and Systems, 2005. (IROS 2005). 2005 IEEE/RSJ International Conference on, 2015.

[4] James Pataleone. Synchronization of metronomes. American Journal of Physics, 2002.

[5] Strogatz and Ian Stewart. Coupled oscillators and biological synchronization. Scientific American, 1993.

[6] Issartel, Marin, and Cadopi. Unintended interpersonal co-ordination: "can we march to the beat of our own drum?". Neurosci Lett. 2007 Jan 16;411(3):174-, 2007.

[7] J. Platkiewiez. Vers une dynamique de coordination coollective: la synchronisation des applaudissements. 2005.

[8] Bernieri and Rosenthal. Fundamentals of non verbal behaviour, p 401-432. R.S Feldman and B. Rime, 1991.

[9] Michael J. Richardson, Kerry L. Marsh, Robert W. Isenhower, Justin R.L. Goodman, and R.C. Schmidt. Rocking together: Dynamics of intentional and unintentional interpersonal coordination. Human Movement Science, 26(6):867 - 891, 2007.

[10] Paula Fiztpatrick, Teresa Mitchell, Jean Frazier, and Richard Schmidt. Exploring the behavioral neural processes underlying social synchronization of inidviduals with and without social deficits, Studies in perception and actions XIII, p40-44. Julie A. Weast-Knapp and MaryLauren, 2015.

[11] Richardson, Rick, Dale, and Kirkhal. the art of conversation is coordination. Apsychological science vol 18 number 5, 2007.

[12] Chloè Leclère ans Sylvie Viaux, Marie Avril, Catherine Achar, Mohamed Chetouani, Sylvain Missonnier, and David Cohen. Why synchrony matters during mother-child interactions: A systematic review. Ed. Sharon Dekel. PLoS ONE 9.12 (2014): e113571. PMC. Web. 9 Sept. 2015., 2014.

[13] FrankJ. Bernieri. Coordinated movement and rapport in teacher-student interactions. Journal of Nonverbal Behavior, 12(2):120-138, 1988. 
[14] Zong Zhao, Robin Salesse, Mathieu Gueugnon, and Richard Schmidt. How you look like matters : impact of physical attractivness on inetrpersonal coordination, Studies in perception and actions XIII, p87-90. Julie A. Weast-Knapp and MaryLauren, 2015.

[15] H. Ogawa and T. Watanabe. Interrobot: a speech driven embodied interaction robot. pages $322-327$, 2000.

[16] Daisuke Sakamoto, Takayuki Kanda, Tetsuo Ono, Masayuki Kamashima, Michita Imai, and Hiroshi Ishiguro. Cooperative embodied communication emerged by interactive humanoid robots. International Journal of Human-Computer Studies, 62(2):247 - 265, 2005. Subtle expressivity for characters and robots.

[17] Manuel Varlet, Ludovic Marin, StÃlphane Raffard, R. C. Schmidt, Delphine Capdevielle, Jean-Philippe Boulenger, Jonathan Del-Monte, and BenoÃöt G. Bardy. Impairments of social motor coordination in schizophrenia. PLoS ONE, 7, 012012.

[18] Kyongsik Yun, Kastumo Watanabe, and Shinsuke Shimojo. Interpersonal body and neural synchronization as a marker of implicit social interation. Scientific Reports - 2:959 -, 2012.

[19] Shinya Fujii, Masaya Hirashima, Kazutoshi Kudo, Tatsuyuki Ohtsuki, Yoshihiko Nakamura, and Shingo Oda. Synchronization error of drum kit playing with a metronome at different tempi by professional drummers. Music Perception: An Interdisciplinary Journal, 28(5):491-503, 2011.

[20] Joanne Lumsden, Lynden K. Miles, and C. Neil Macrae. Sync or sink? interpersonal synchrony impacts self-esteem. Frontiers in Psychology, 5:1064, 2014.

[21] T. Lorenz, A. Mörtl, and S. Hirche. Movement synchronization fails during non-adaptive human-robot interaction. In 2013 8th ACM/IEEE International Conference on Human-Robot Interaction (HRI), pages 189-190, March 2013.

[22] G Dumas and R Soussignan J Nadel. Inter-brain synchronization during social interaction. PLos ONE $5(8), 2010$.

[23] J. A. Scott Kelso. Haken-Kelso-Bunz model. Scholarpedia, 3(10):1612, 2008. revision 91336.

[24] H.R Wilson and J.D Cowan J. Excitatory and inhibitory interactions in localized populations of model neurons. Biophys. J., Vol. 12, 1972.

[25] Syed Khursheed Hasnain, Ghiles Mostafaoui, and Philippe Gaussier. A synchrony-based perspective for partner selection and attentional mechanism in human-robot interaction. Paladyn Journal of Behavioral Robotics, 3(3):156-171, 2012.

[26] Kena Prepin and Philippe Gaussier. How an Agent Can Detect and Use Synchrony Parameter of Its Own Interaction with a Human?, pages 50-65. Springer Berlin Heidelberg, Berlin, Heidelberg, 2010.

[27] Tomer Amiaz, Eyal Lubetzky, and Nahum Kiryati. Coarse to over-fine optical flow estimation. Pattern Recogn., 40(9):2496-2503, September 2007. 